\title{
The Design of the Parallel Box Cavity with New Radial RF Coupler Design
}

\section{Al Moretti}

Induction

Previous studies of the current LBL Pillbox cavity have shown a large amount of sparking damage occurred in the RF coupler region of the cavity and a low sparking rate gradient limit of $16 \mathrm{MV} / \mathrm{m}$ in a parallel $3 \mathrm{~T} \mathrm{DC}$ magnetic field. It is suspected that the reason for this is that the coupler is not inserted on the minimum electric field surface and therefore, has a large enhancement of the electric field on the coupling aperture. Studies of the LBL Pillbox cavity have shown that the coupling aperture is the location of the highest gradient throughout the cavity for reasonable aperture radii. The report describes a design that moves the RF coupler aperture to a radial position a surface of minimum electric field gradient, Figure 1 . The narrow sides of the box are all surface of minimum electric field gradient, and it is shown to achieve a minimum enhancement of the gradient of the coupling aperture even with relatively small aperture radius.

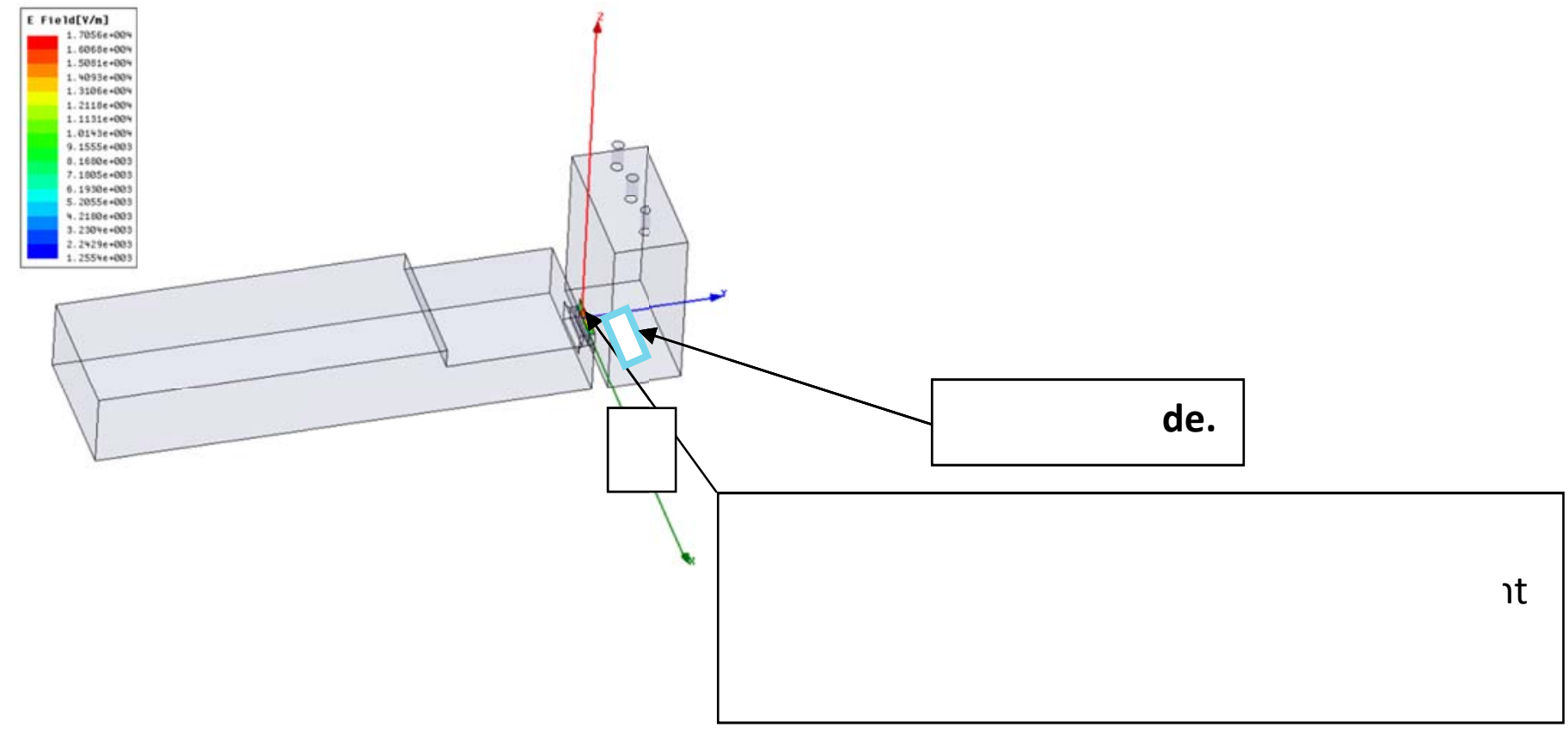


Another reason for this change is that it corresponds to the location of the coupling aperture in a future real experimental cooling channel. Also, the criticism will occur that the real cavity design has not been tested. The collaboration may then be forced to test this type of cavity design. Also, for this design the coupling factor to the cavity is adjustable externally, a very useful feature.

\section{The Old Design Effort}

Tim Hamerla has completed the mechanical design of the "Old" parallel E/H box cavity, drawings \#'s 2130-000-MB-391363 to 391370, Figure 2. The coupling aperture needs to be move to the side as shown for the "New design" in Figure 1. Figure 3 show more details of the current parallel box cavity design. The original LBL RF power coupling waveguide section will stay the same and be reused for high RF power coupling into the cavity. 


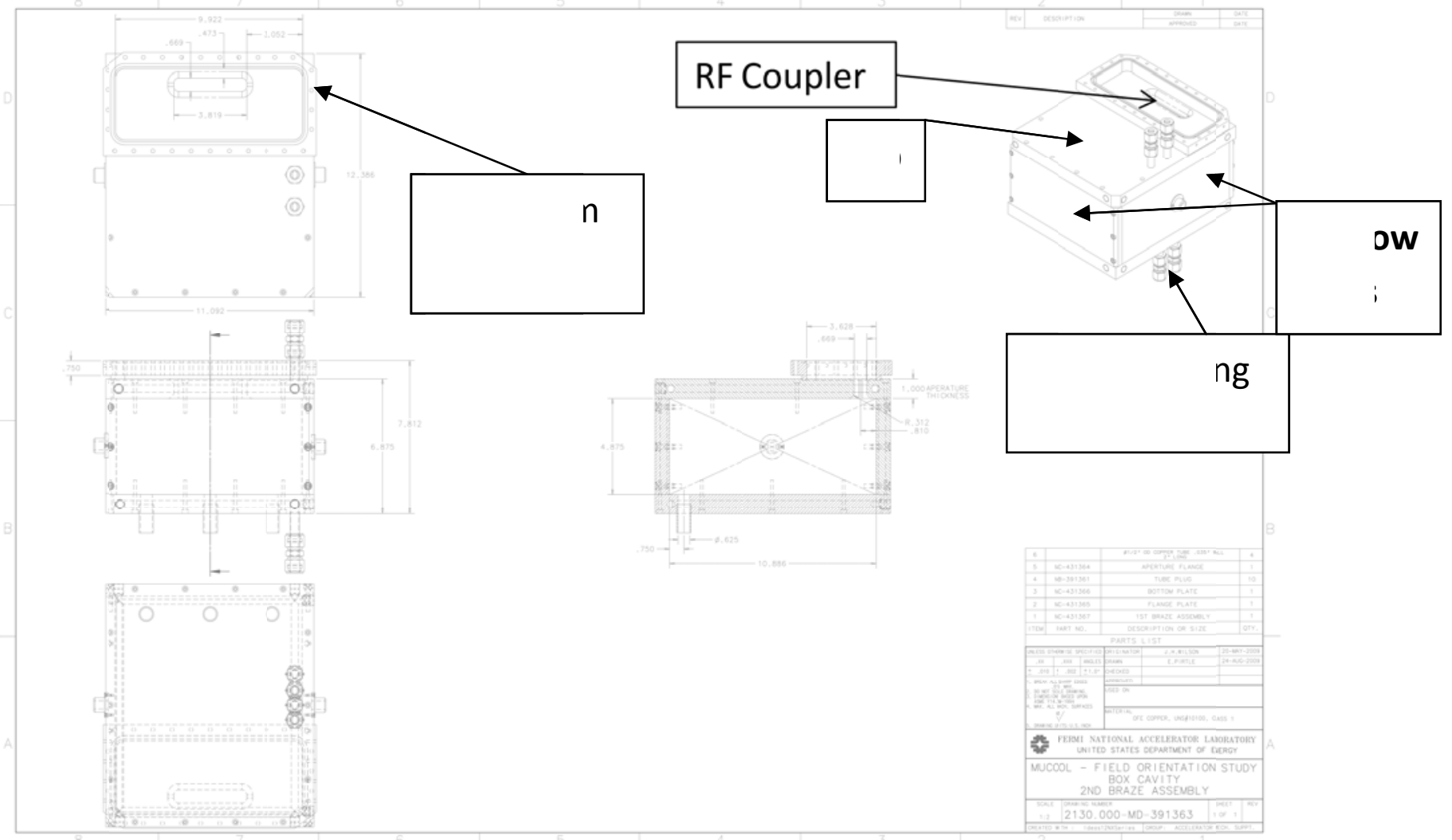

The design shows the construction of the cavity from brazed together OFE copper plates 1 inch thick. It takes $\mathbf{2}$ hydrogen oven brazing cycles to complete the cavity. The first cycle brazes together the 4 sides to make an open box. The second cycles brazes the top plate with its attachments and the bottom plate to the open box to complete the fabrication. The RF carrying surfaces ( the insides surfaces of the cavity) are to be machined to a finish of $10 \mu$ inches. 
Figure 4. Details of the current cavity support structure in the magnet.

As shown in Figure 4 the cavity is supported by an aluminum frame bolted to the support frame of the magnet. It is first assembled on to the Transport carriage in a cleanroom, attached to RF power coupler, short coupling cell and vacuum checked. It is then moved to the MTA hall and inserted into the magnet. There is a set of rollers and rails that allow this to be done easily. The difference in the "New design" is that coupling aperture will be moved to the bottom narrow side. This will require the majority of the redesign effort because the coupling waveguide aperture is being moved the bottom. The box cavity itself will requires minimum design changes.

The "New Parallel" box cavity design details

Figure $\mathbf{5} \mathrm{a}$ and $\mathrm{b}$ shows details of the HFSS simulation program of the "New design" with the coupling cell coming in from the bottom of the cavity. The HFSS program produces dimensional mapping of the cavity suitable for detailed mechanical design of its parts. The dashed area in Figure $5 \mathrm{a}$, shows the location of the coupling cell that will require the major redesign effort.

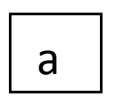




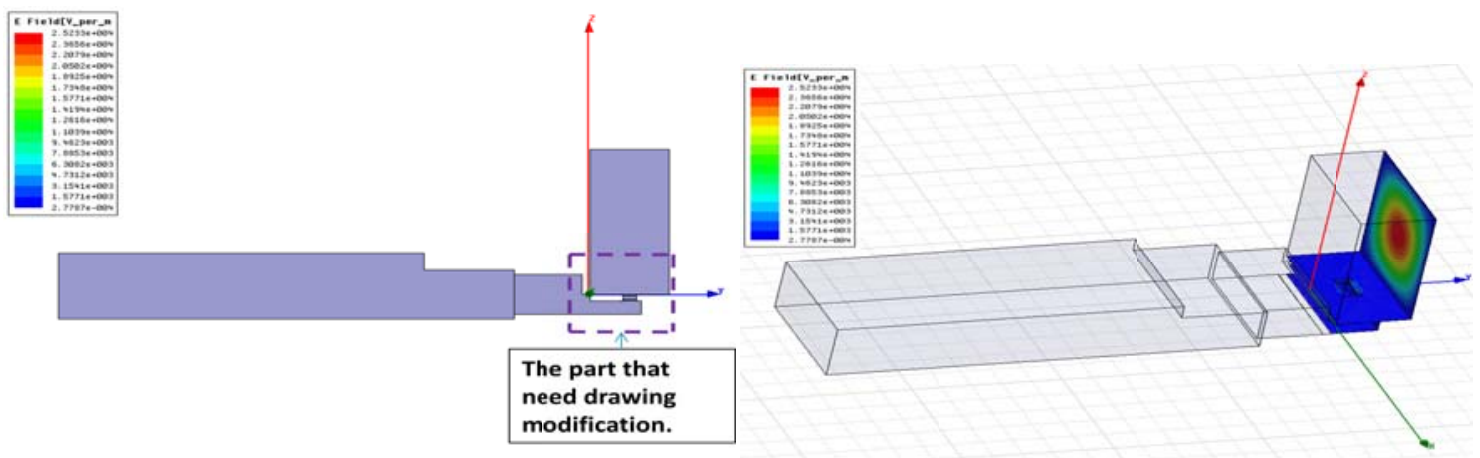

Figure 5 a and b show details of the "New Parallel E/H" Box Cavity Design.
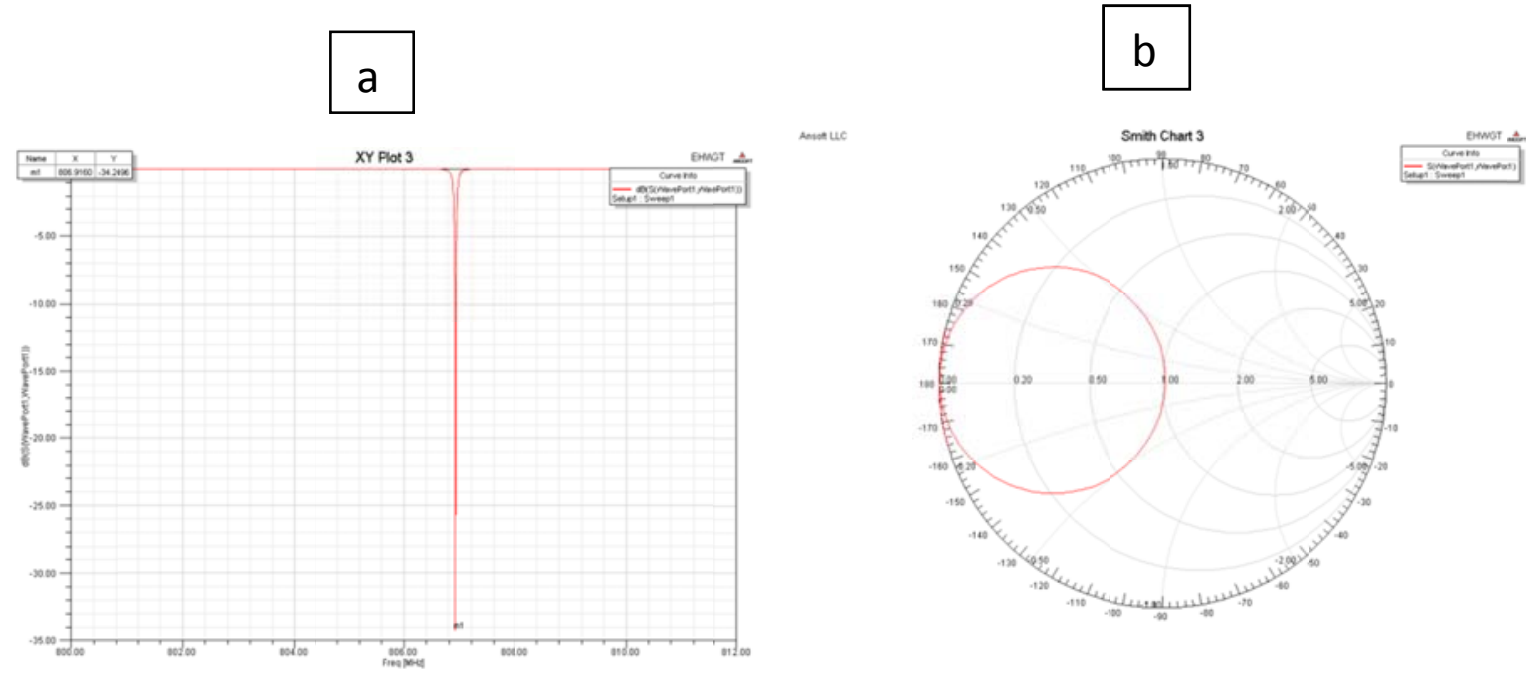

Figure $6 a$ and $b$ show the HFSS RF characteristics simulation of the cavity

By designing the waveguide coupler end as an adjustable external short, Figure $5 \mathrm{a}$, the coupling factor can be made broadly adjustable. For example, linac cavities are normally set to be overcoupled to match the expected beam loading. This will allow broad coupling matching for all beam condition experienced over time. Figure $\mathbf{5} \mathbf{b}$ top view of the coupler, demonstrates that there is little or no enhancement of the electric field on the coupling aperture, dark blue area.

Figure $6 a$ and 6 b demonstrates the RF characteristics of the HFSS simulation of the cavity design. It shows a resonant frequency of $806.916 \mathrm{MHz}$ and that it is a well matched design. The resonant frequency is well within the bandwidth of 
the high power final driver klystron. The HFSS program has been very successful in predicting the performance of the cavity machined to its dimensions. No further manual tuning was required to meet the simulation specification, the orthogonal box cavity. Normally with previous programs further manual tuning is required to meet the specifications.

\section{Summary}

The design of a E/H parallel cavity has been described. It has been designed with the RF simulation program HFSS. HFSS has been very successfully been used to design and fabricate the Orthogonal $\mathrm{E} / \mathrm{H}$ box cavity. The main reason for this design choice is to move the RF coupling aperture to surface of minimum electric field gradient. It is suspected that this change will remove the damaged observed in the coupling area experimentally observed in the LBL Pillbox cavity. Another reason for this change is that it corresponds to the location of the coupling aperture in a future real experimental cooling channel. Also, with this design the coupling factor to the cavity can be made adjustable externally, a very useful feature. 